DESY 04-032

ISSN 0418-9833

March 2004

\title{
Measurement of Anti-Deuteron Photoproduction and a Search for Heavy Stable Charged Particles at HERA
}

\author{
H1 Collaboration
}

\begin{abstract}
The cross section for anti-deuteron photoproduction is measured at HERA at a mean centreof-mass energy of $W_{\gamma p}=200 \mathrm{GeV}$ in the range $0.2<p_{T} / M<0.7$ and $|y|<0.4$, where $M, p_{T}$ and $y$ are the mass, transverse momentum and rapidity in the laboratory frame of the anti-deuteron, respectively. The numbers of anti-deuterons per event are found to be similar in photoproduction to those in central proton-proton collisions at the CERN ISR but much lower than those in central Au-Au collisions at RHIC. The coalescence parameter $B_{2}$, which characterizes the likelihood of anti-deuteron production, is measured in photoproduction to be $0.010 \pm 0.002 \pm 0.001$, which is much higher than in Au-Au collisions at a similar nucleon-nucleon centre-of-mass energy. No significant production of particles heavier than deuterons is observed and upper limits are set on the photoproduction cross sections for such particles.
\end{abstract}

(Submitted to European Physical Journal C) 
A. Aktas ${ }^{10}$, V. Andreev ${ }^{26}$, T. Anthonis ${ }^{4}$, A. Asmone ${ }^{33}$, A. Babaev ${ }^{25}$, S. Backovic ${ }^{37}$, J. Bährr ${ }^{37}$, P. Baranov ${ }^{26}$, E. Barrelet ${ }^{30}$, W. Bartel ${ }^{10}$, S. Baumgartner ${ }^{38}$, J. Becker ${ }^{39}$, M. Beckingham ${ }^{21}$, O. Behnke ${ }^{13}$, O. Behrendt ${ }^{7}$, A. Belousov ${ }^{26}$, Ch. Berger ${ }^{1}$, N. Berger ${ }^{38}$, T. Berndt ${ }^{14}$, J.C. Bizot ${ }^{28}$, J. Böhme ${ }^{10}$, M.-O. Boenig ${ }^{7}$, V. Boudry ${ }^{29}$, J. Bracinik ${ }^{27}$, V. Brisson ${ }^{28}$, H.-B. Bröker ${ }^{2}$, D.P. Brown ${ }^{10}$, D. Bruncko ${ }^{16}$, F.W. Büsser ${ }^{11}$, A. Bunyatyan ${ }^{12,36}$, G. Buschhorn ${ }^{27}$, L. Bystritskaya ${ }^{25}$, A.J. Campbell ${ }^{10}$, S. Caron ${ }^{1}$, F. Cassol-Brunner ${ }^{22}$, K. Cerny ${ }^{32}$, V. Chekelian ${ }^{27}$, C. Collard ${ }^{4}$, J.G. Contreras ${ }^{23}$, Y.R. Coppens ${ }^{3}$, J.A. Coughlan ${ }^{5}$, B.E. Cox ${ }^{21}$, G. Cozzika ${ }^{9}$, J. Cvach ${ }^{31}$, J.B. Dainton ${ }^{18}$, W.D. Dau ${ }^{15}$, K. Daum ${ }^{35,41}$, B. Delcourt ${ }^{28}$, R. Demirchyan ${ }^{36}$, A. De Roeck ${ }^{10,44}$, K. Desch ${ }^{11}$, E.A. De Wolf ${ }^{4}$, C. Diaconu ${ }^{22}$, J. Dingfelder ${ }^{13}$, V. Dodonov ${ }^{12}$, A. Dubak ${ }^{27}$, C. Duprel ${ }^{2}$, G. Eckerlin ${ }^{10}$, V. Efremenko ${ }^{25}$, S. Egli ${ }^{34}$, R. Eichler $^{34}$, F. Eisele ${ }^{13}$, M. Ellerbrock ${ }^{13}$, E. Elsen ${ }^{10}$, M. Erdmann ${ }^{10,42}$, W. Erdmann ${ }^{38}$, P.J.W. Faulkner ${ }^{3}$, L. Favart ${ }^{4}$, A. Fedotov ${ }^{25}$, R. Felst ${ }^{10}$, J. Ferencei ${ }^{10}$, M. Fleischer ${ }^{10}$, P. Fleischmann ${ }^{10}$, Y.H. Fleming ${ }^{10}$, G. Flucke ${ }^{10}$, G. Flügge ${ }^{2}$, A. Fomenko ${ }^{26}$, I. Foresti ${ }^{39}$, J. Formánek ${ }^{32}$, G. Franke ${ }^{10}$, G. Frising ${ }^{1}$, E. Gabathuler ${ }^{18}$, K. Gabathuler ${ }^{34}$, E. Garutti ${ }^{10}$, J. Garvey ${ }^{3}$, J. Gayler ${ }^{10}$, R. Gerhards ${ }^{10 \dagger}$, C. Gerlich ${ }^{13}$, S. Ghazaryan ${ }^{36}$, L. Goerlich ${ }^{6}$, N. Gogitidze ${ }^{26}$, S. Gorbounov ${ }^{37}$, C. Grab $^{38}$, H. Grässler ${ }^{2}$, T. Greenshaw ${ }^{18}$, M. Gregori ${ }^{19}$, G. Grindhammer ${ }^{27}$, C. Gwilliam ${ }^{21}$, D. Haidt ${ }^{10}$, L. Hajduk ${ }^{6}$, J. Haller ${ }^{13}$, M. Hansson ${ }^{20}$, G. Heinzelmann ${ }^{11}$, R.C.W. Henderson ${ }^{17}$, H. Henschel ${ }^{37}$, O. Henshaw ${ }^{3}$, R. Heremans ${ }^{4}$, G. Herrera ${ }^{24}$, I. Herynek ${ }^{31}$, R.-D. Heuer ${ }^{11}$, M. Hildebrandt ${ }^{34}$, K.H. Hiller ${ }^{37}$, P. Höting ${ }^{2}$, D. Hoffmann ${ }^{22}$, R. Horisberger ${ }^{34}$, A. Hovhannisyan ${ }^{36}$, M. Ibbotson ${ }^{21}$, M. Ismail ${ }^{21}$, M. Jacquet ${ }^{28}$, L. Janauschek ${ }^{27}$, X. Janssen ${ }^{10}$, V. Jemanov ${ }^{11}$, L. Jönsson ${ }^{20}$, D.P. Johnson ${ }^{4}$, H. Jung ${ }^{20,10}$, D. Kant ${ }^{19}$, M. Kapichine ${ }^{8}$, M. Karlsson ${ }^{20}$, J. Katzy ${ }^{10}$, N. Keller ${ }^{39}$, J. Kennedy ${ }^{18}$, I.R. Kenyon ${ }^{3}$, C. Kiesling ${ }^{27}$, M. Klein ${ }^{37}$, C. Kleinwort ${ }^{10}$, T. Klimkovich ${ }^{10}$, T. Kluge ${ }^{1}$, G. Knies ${ }^{10}$, A. Knutsson ${ }^{20}$, B. Koblitz ${ }^{27}$, V. Korbel ${ }^{10}$, P. Kostka ${ }^{37}$, R. Koutouev ${ }^{12}$, A. Kropivnitskaya ${ }^{25}$, J. Kroseberg ${ }^{39}$, J. Kückens ${ }^{10}$, T. Kuhr ${ }^{10}$, M.P.J. Landon ${ }^{19}$, W. Lange ${ }^{37}$, T. Laštovička ${ }^{37,32}$, P. Laycock ${ }^{18}$, A. Lebedev ${ }^{26}$, B. Leißner ${ }^{1}$, R. Lemrani ${ }^{10}$, V. Lendermann ${ }^{14}$, S. Levonian ${ }^{10}$, L. Lindfeld ${ }^{39}$, K. Lipka ${ }^{37}$, B. List ${ }^{38}$, E. Lobodzinska ${ }^{37,6}$, N. Loktionova ${ }^{26}$, R. Lopez-Fernandez ${ }^{10}$, V. Lubimov ${ }^{25}$, H. Lueders ${ }^{11}$, D. Lüke ${ }^{7,10}$, T. Lux ${ }^{11}$, L. Lytkin ${ }^{12}$, A. Makankine ${ }^{8}$, N. Malden ${ }^{21}$, E. Malinovski ${ }^{26}$, S. Mangano ${ }^{38}$, P. Marage ${ }^{4}$, J. Marks ${ }^{13}$, R. Marshall ${ }^{21}$, M. Martisikova ${ }^{10}$, H.-U. Martyn ${ }^{1}$, S.J. Maxfield ${ }^{18}$, D. Meer ${ }^{38}$, A. Mehta ${ }^{18}$, K. Meier ${ }^{14}$, A.B. Meyer ${ }^{11}$, H. Meyer ${ }^{35}$, J. Meyer ${ }^{10}$, S. Michine ${ }^{26}$, S. Mikocki ${ }^{6}$, I. Milcewicz ${ }^{6}$, D. Milstead ${ }^{18}$, A. Mohamed ${ }^{18}$, F. Moreau ${ }^{29}$, A. Morozov ${ }^{8}$, I. Morozov $^{8}$, J.V. Morris ${ }^{5}$, M.U. Mozer ${ }^{13}$, K. Müller ${ }^{39}$, P. Murín ${ }^{16,43}$, V. Nagovizin ${ }^{25}$, B. Naroska ${ }^{11}$, J. Naumann ${ }^{7}$, Th. Naumann ${ }^{37}$, P.R. Newman ${ }^{3}$, C. Niebuhr ${ }^{10}$, A. Nikiforov ${ }^{27}$, D. Nikitin ${ }^{8}$, G. Nowak ${ }^{6}$, M. Nozicka ${ }^{32}$, R. Oganezov ${ }^{36}$, B. Olivier ${ }^{10}$, J.E. Olsson ${ }^{10}$, G.Ossoskov $^{8}$, D. Ozerov ${ }^{25}$, C. Pascaud $^{28}$, G.D. Patel ${ }^{18}$, M. Peez ${ }^{29}$, E. Perez ${ }^{9}$, A. Perieanu ${ }^{10}$, A. Petrukhin ${ }^{25}$, D. Pitzl ${ }^{10}$, R. Plačakytè ${ }^{27}$, R. Pöschl ${ }^{10}$, B. Portheault ${ }^{28}$, B. Povh ${ }^{12}$, N. Raicevic ${ }^{37}$, Z. Ratiani ${ }^{10}$, P. Reimer ${ }^{31}$, B. Reisert ${ }^{27}$, A. Rimmer ${ }^{18}$, C. Risler ${ }^{27}$, E. Rizvi ${ }^{3}$, P. Robmann ${ }^{39}$, B. Roland ${ }^{4}$, R. Roosen ${ }^{4}$, A. Rostovtsev ${ }^{25}$, Z. Rurikova ${ }^{27}$, S. Rusakov ${ }^{26}$, K. Rybicki ${ }^{6, \dagger}$, D.P.C. Sankey ${ }^{5}$, E. Sauvan ${ }^{22}$, S. Schätzel ${ }^{13}$, J. Scheins ${ }^{10}$, F.-P. Schilling ${ }^{10}$, P. Schleper ${ }^{10}$, S. Schmidt ${ }^{27}$, S. Schmitt ${ }^{39}$, M. Schneider ${ }^{22}$, L. Schoeffel ${ }^{9}$, A. Schöning ${ }^{38}$, V. Schröder ${ }^{10}$, H.-C. Schultz-Coulon ${ }^{14}$, C. Schwanenberger ${ }^{10}$, K. Sedlák ${ }^{31}$, F. Sefkow ${ }^{10}$, I. Sheviakov ${ }^{26}$, L.N. Shtarkov ${ }^{26}$, Y. Sirois ${ }^{29}$, T. Sloan ${ }^{17}$, P. Smirnov ${ }^{26}$, Y. Soloviev ${ }^{26}$, D. South ${ }^{10}$, V. Spaskov ${ }^{8}$, A. Specka ${ }^{29}$, H. Spitzer ${ }^{11}$, R. Stamen ${ }^{10}$, B. Stella ${ }^{33}$, J. Stiewe ${ }^{14}$, I. Strauch ${ }^{10}$, U. Straumann ${ }^{39}$, V. Tchoulakov ${ }^{8}$, G. Thompson ${ }^{19}$, P.D. Thompson ${ }^{3}$, F. Tomasz ${ }^{14}$, D. Traynor ${ }^{19}$, P. Truöl ${ }^{39}$, G. Tsipolitis ${ }^{10,40}$, I. Tsurin ${ }^{37}$, J. Turnau ${ }^{6}$, E. Tzamariudaki ${ }^{27}$, A. Uraev ${ }^{25}$, M. Urban ${ }^{39}$, 
A. Usik ${ }^{26}$, D. Utkin ${ }^{25}$, S. Valkár ${ }^{32}$, A. Valkárováa ${ }^{32}$, C. Vallée ${ }^{22}$, P. Van Mechelen ${ }^{4}$, N. Van

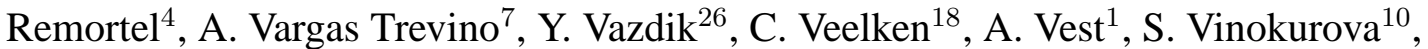
V. Volchinski ${ }^{36}$, K. Wacker ${ }^{7}$, J. Wagner ${ }^{10}$, G. Weber ${ }^{11}$, R. Weber ${ }^{38}$, D. Wegener ${ }^{7}$, C. Werner ${ }^{13}$, N. Werner ${ }^{39}$, M. Wessels ${ }^{1}$, B. Wessling ${ }^{11}$, G.-G. Winter ${ }^{10}$, Ch. Wissing ${ }^{7}$, E.-E. Woehrling ${ }^{3}$, R. Wolf ${ }^{13}$, E. Wünsch ${ }^{10}$, S. Xella ${ }^{39}$, W. Yan ${ }^{10}$, V. Yeganov ${ }^{36}$, J. Žáček ${ }^{32}$, J. Zálešák ${ }^{31}$, Z. Zhang ${ }^{28}$, A. Zhokin ${ }^{25}$, H. Zohrabyan ${ }^{36}$, and F. Zomer ${ }^{28}$

${ }^{1}$ I. Physikalisches Institut der RWTH, Aachen, Germany ${ }^{a}$

${ }^{2}$ III. Physikalisches Institut der RWTH, Aachen, Germany ${ }^{a}$

${ }^{3}$ School of Physics and Space Research, University of Birmingham, Birmingham, $U K^{b}$

${ }^{4}$ Inter-University Institute for High Energies ULB-VUB, Brussels; Universiteit Antwerpen (UIA), Antwerpen; Belgium ${ }^{c}$

${ }^{5}$ Rutherford Appleton Laboratory, Chilton, Didcot, $U K^{b}$

${ }^{6}$ Institute for Nuclear Physics, Cracow, Poland ${ }^{d}$

${ }^{7}$ Institut für Physik, Universität Dortmund, Dortmund, Germany ${ }^{a}$

${ }^{8}$ Joint Institute for Nuclear Research, Dubna, Russia

${ }^{9}$ CEA, DSM/DAPNIA, CE-Saclay, Gif-sur-Yvette, France

${ }^{10}$ DESY, Hamburg, Germany

${ }^{11}$ Institut für Experimentalphysik, Universität Hamburg, Hamburg, Germany ${ }^{a}$

${ }^{12}$ Max-Planck-Institut für Kernphysik, Heidelberg, Germany

${ }^{13}$ Physikalisches Institut, Universität Heidelberg, Heidelberg, Germany ${ }^{a}$

${ }^{14}$ Kirchhoff-Institut für Physik, Universität Heidelberg, Heidelberg, Germany ${ }^{a}$

${ }^{15}$ Institut für experimentelle und Angewandte Physik, Universität Kiel, Kiel, Germany

${ }^{16}$ Institute of Experimental Physics, Slovak Academy of Sciences, Košice, Slovak Republic e,f

${ }^{17}$ Department of Physics, University of Lancaster, Lancaster, $U K^{b}$

${ }^{18}$ Department of Physics, University of Liverpool, Liverpool, $U K^{b}$

${ }^{19}$ Queen Mary and Westfield College, London, $U K^{b}$

${ }^{20}$ Physics Department, University of Lund, Lund, Sweden ${ }^{g}$

${ }^{21}$ Physics Department, University of Manchester, Manchester, $U K^{b}$

${ }^{22}$ CPPM, CNRS/IN2P3 - Univ Mediterranee, Marseille - France

${ }^{23}$ Departamento de Fisica Aplicada, CINVESTAV, Mérida, Yucatán, México ${ }^{k}$

${ }^{24}$ Departamento de Fisica, CINVESTAV, México ${ }^{k}$

${ }^{25}$ Institute for Theoretical and Experimental Physics, Moscow, Russial

${ }^{26}$ Lebedev Physical Institute, Moscow, Russia ${ }^{e}$

${ }^{27}$ Max-Planck-Institut für Physik, München, Germany

${ }^{28}$ LAL, Université de Paris-Sud, IN2P3-CNRS, Orsay, France

${ }^{29}$ LLR, Ecole Polytechnique, IN2P3-CNRS, Palaiseau, France

${ }^{30}$ LPNHE, Universités Paris VI and VII, IN2P3-CNRS, Paris, France

${ }^{31}$ Institute of Physics, Academy of Sciences of the Czech Republic, Praha, Czech Republic ${ }^{e, i}$

${ }^{32}$ Faculty of Mathematics and Physics, Charles University, Praha, Czech Republic e,i

${ }^{33}$ Dipartimento di Fisica Università di Roma Tre and INFN Roma 3, Roma, Italy

${ }^{34}$ Paul Scherrer Institut, Villigen, Switzerland

${ }^{35}$ Fachbereich Physik, Bergische Universität Gesamthochschule Wuppertal, Wuppertal, Germany

${ }^{36}$ Yerevan Physics Institute, Yerevan, Armenia

${ }^{37}$ DESY, Zeuthen, Germany 
${ }^{38}$ Institut für Teilchenphysik, ETH, Zürich, Switzerland ${ }^{j}$

${ }^{39}$ Physik-Institut der Universität Zürich, Zürich, Switzerland ${ }^{j}$

${ }^{40}$ Also at Physics Department, National Technical University, Zografou Campus, GR-15773 Athens, Greece

${ }^{41}$ Also at Rechenzentrum, Bergische Universität Gesamthochschule Wuppertal, Germany

${ }^{42}$ Also at Institut für Experimentelle Kernphysik, Universität Karlsruhe, Karlsruhe, Germany

${ }^{43}$ Also at University of P.J. Šafárik, Košice, Slovak Republic

${ }^{44}$ Also at CERN, Geneva, Switzerland

$\dagger$ Deceased

${ }^{a}$ Supported by the Bundesministerium für Bildung und Forschung, FRG, under contract numbers 05 H1 1GUA /1, 05 HI 1PAA /1, 05 H1 1PAB /9, 05 H1 1PEA /6, 05 H1 1VHA /7 and $05 \mathrm{HI} 1 \mathrm{VHB} / 5$

${ }^{b}$ Supported by the UK Particle Physics and Astronomy Research Council, and formerly by the UK Science and Engineering Research Council

${ }^{c}$ Supported by FNRS-FWO-Vlaanderen, IISN-IIKW and IWT

${ }^{d}$ Partially Supported by the Polish State Committee for Scientific Research, SPUB/DESY/P003/DZ 118/2003/2005

e Supported by the Deutsche Forschungsgemeinschaft

${ }^{f}$ Supported by VEGA SR grant no. 2/1169/2001

${ }^{g}$ Supported by the Swedish Natural Science Research Council

${ }^{i}$ Supported by the Ministry of Education of the Czech Republic under the projects INGO-LA116/2000 and LNOOA006, by GAUK grant no 173/2000

${ }^{j}$ Supported by the Swiss National Science Foundation

${ }^{k}$ Supported by CONACYT, México, grant 400073-F

${ }^{l}$ Partially Supported by Russian Foundation for Basic Research, grant no. 00-15-96584 


\section{Introduction}

This paper describes a measurement of the rate of production of anti-deuterons in photon-proton collisions at HERA performed by the $\mathrm{H} 1$ Collaboration. The measurement is of particular interest in the context of recent studies of heavy ion collisions [1]. In these collisions, the deuteron and anti-deuteron production rate is thought to depend on the dimensions of the collision "fireball" at the stage at which the hadrons decouple [2], i.e. when final state interactions become unimportant. This is the so-called "thermal freeze-out" region.

Further, a search is performed for the photoproduction of unknown charged stable heavy particles in the highest energy electron-proton collisions currently accessible in the laboratory. The discovery of such particles would be an indication for physics beyond the Standard Model.

The production of nuclei in particle collisions can be described in terms of the coalescence model. In this model [3], the cross section, $\sigma_{A}$, for the formation of an object with $A$ nucleons with total energy $E_{A}$ and momentum $P$, is related to that for the production of free nucleons in the same reaction, $\sigma_{N}$, with energy $E_{N}$ and momentum $p=P / A$, by

$$
\frac{1}{\sigma} \frac{E_{A} \mathrm{~d}^{3} \sigma_{\mathrm{A}}}{\mathrm{d}^{3} \mathrm{P}}=B_{A}\left(\frac{1}{\sigma} \frac{E_{N} \mathrm{~d}^{3} \sigma_{\mathrm{N}}}{\mathrm{d}^{3} \mathrm{p}}\right)^{A},
$$

where $B_{A}$ is the coalescence parameter, which is inversely proportional to the source volume in heavy ion collisions [2], and $\sigma$ is the total interaction cross section of the colliding particles.

For the measurements described here, the particles are identified through a combination of their specific ionisation energy loss, $\mathrm{d} E / \mathrm{d} x$, and their momenta. The numbers of anti-deuterons are measured in the laboratory frame in the rapidity region $|y|<0.4$ which corresponds to a centre-of-mass rapidity of between 1.6 and 2.4 units $^{1}$. In this range, the multiplicity distributions are on the central plateau [4] and so comparisons can reasonably be made with the measured numbers of anti-deuterons per event in central proton-proton collisions $[5,6]$. Both are contrasted with data from heavy ion collisions $[7,8]$. These comparisons are restricted to central collisions at a centre-of-mass energy greater than $50 \mathrm{GeV}$, i.e. well above the threshold for anti-deuteron production. There have been several other measurements of anti-deuteron production in proton-proton $(p p)$ [9], proton-nucleus $(p A)$ [10], nucleus-nucleus $(A A)$ [8] and electron-positron [11] collisions. These measurements are either for non-central production or are at a centre-of-mass energy below $50 \mathrm{GeV}$.

\section{Experimental Procedure}

\subsection{The H1 Detector}

Collisions of $27.6 \mathrm{GeV}$ positrons with $820 \mathrm{GeV}$ protons at HERA are detected in the $\mathrm{H} 1$ detector, which is described in detail elsewhere [12]. The components of the detector important in

\footnotetext{
${ }^{1}$ The rapidity and pseudorapidity are defi ned by $y=0.5 \ln \left[\left(E+p_{z}\right) /\left(E-p_{z}\right)\right]$ and $\eta=-\ln (\tan \theta / 2)$ for a particle with total energy $E, z$ component of momentum $p_{z}$ and polar angle $\theta$. The $+z$-axis (forward direction) is taken to be along the proton beam direction.
} 
this analysis are the small angle positron tagger, the central tracker, the backward Spaghetti-type calorimeter (SpaCal) and the liquid argon (LAr) calorimeter.

The positron tagger, located at $33 \mathrm{~m}$ from the interaction point in the outgoing positron beam direction, is used to trigger on photoproduction events and to measure the energy of the scattered positron, from which the total photon-proton centre-of-mass energy, $W_{\gamma p}$, is deduced. The central track detector, surrounding the $9 \mathrm{~cm}$ diameter aluminium beam pipe of thickness $1.7 \mathrm{~mm}$, consists of concentric central jet drift chambers (CJCs) with inner (CJC1) and outer (CJC2) chambers and two additional drift chambers which measure the $z$ coordinates of tracks. The pseudorapidity range covered by the central track detector is $|\eta|<1.5$. The CJC has 56 sensitive wire layers: 24 in CJC1 covering radii from $20.3 \mathrm{~cm}$ to $45.1 \mathrm{~cm}$ and 32 in CJC2 covering radii from $53.0 \mathrm{~cm}$ to $84.4 \mathrm{~cm}$. The detector is placed inside a uniform magnetic field of $1.15 \mathrm{~T}$, allowing measurements of the track transverse momentum to be made with a resolution of $\sigma_{p_{T}} / p_{T} \approx 0.009 \cdot p_{T}[\mathrm{GeV}] \oplus 0.015$. The specific energy loss, $\mathrm{d} E / \mathrm{d} x$, of the charged particles is also measured in this detector with a resolution $\sigma(\mathrm{d} E / \mathrm{d} x) /(\mathrm{d} E / \mathrm{d} x)$ of $7.5 \%$ for 56 hits on a minimum ionising track. The LAr calorimeter covers the angular range $4^{\circ}<\theta<154^{\circ}$ with the forward region defined to be $4^{\circ}<\theta<25^{\circ}$. The SpaCal calorimeter covers the backward region, i.e. the angular range $153^{\circ}<\theta<177.8^{\circ}$. The luminosity is measured via the well understood Bethe-Heitler process, $e p \rightarrow e p \gamma$, using a photon detector at $0^{\circ}$ to the positron beam direction.

\subsection{Trigger Conditions, Event and Track Selection}

The measurements presented here are based on $\mathrm{H} 1$ data taken with minimally biased triggers in 1996 and correspond to an integrated luminosity of $5.53 \pm 0.11 \mathrm{pb}^{-1}$. Photoproduction events are triggered by requiring the presence of tracks in the $\mathrm{CJC}$ and of a scattered positron in the positron tagger, which ensures that the photon virtuality $Q^{2}<10^{-2} \mathrm{GeV}^{2}$. The following selection criteria are applied in order to reduce the background contamination and to ensure good reconstruction of the event kinematics. The selected events are required to lie within the interval $165<W_{\gamma p}<252 \mathrm{GeV}$ (average $\left\langle W_{\gamma p}\right\rangle=200 \mathrm{GeV}$ ). In this range the total acceptance of the positron tagger for photoproduction events is $0.46 \pm 0.02$. In addition, five or more tracks are required to be reconstructed in the CJC. These tracks must point to a common vertex with $z$ coordinate within $\pm 30 \mathrm{~cm}$ of the nominal interaction point.

Candidate tracks for particle identification are selected in the range $|\eta|<1$ so that they are well contained within the CJC. Here the track reconstruction and particle identification efficiencies are high. Two track selection schemes are used: the "hard" and "soft" selections. The minimum ionising particle (MIP) background is larger in the soft selection scheme than in the hard selection scheme. The hard selection is used to search for rare heavy particles, when it is necessary to minimise this background and to have optimum $\mathrm{d} E / \mathrm{d} x$ resolution. The soft selection is used only for copiously produced particles such as protons and anti-protons.

In the soft selection, the tracks are required to have at least 10 hits and to have a start point at radius $<30 \mathrm{~cm}$ from the beam line and an end point at radius $>37.5 \mathrm{~cm}$. The total measured radial track length is required to be more than $10 \mathrm{~cm}$ and the specific energy loss to be more 
than twice that of a MIP, i.e. $\log _{10} \mathrm{~d} E / \mathrm{d} x>0.3 .^{2}$ These criteria are loose enough to ensure that the combined track reconstruction and particle identification efficiency is high (measured to be $98.8 \pm 0.2 \%$ ). In the hard selection, tracks are required to pass through both CJC1 and CJC2. The number of hits in CJC1 is required to be at least 20 with a minimum of 40 hits in both chambers. The total measured radial track length must be greater than $35 \mathrm{~cm}$. In addition, the selected tracks must have a minimum of $75 \%$ of the total number of possible hits and a specific energy loss of more than 2.5 times that of a MIP, i.e. $\log _{10}(\mathrm{~d} E / \mathrm{d} x)>0.4$.

\subsection{Particle Identification.}

The mass, $M$, associated with each charged particle track is deduced from the track momentum, $p$, and the most probable specific energy loss, $\mathrm{d} E / \mathrm{d} x_{0}$, which is determined using a Bayesian $\log$-likelihood method $[13,14]$. The value of $\mathrm{d} E / \mathrm{d} x_{0}$ is chosen for each track such that the likelihood function

$$
\log L=\sum_{i}^{N} \log P\left(\mathrm{~d} E / \mathrm{d} x_{i} \mid \mathrm{d} E / \mathrm{d} x_{0}\right)
$$

is maximised. Here, $P\left(\mathrm{~d} E / \mathrm{d} x_{i} \mid \mathrm{d} E / \mathrm{d} x_{0}\right)$ is the probability that the $i^{\text {th }}$ measured value of $\mathrm{d} E / \mathrm{d} x$ results from a particle with most probable specific energy loss $\mathrm{d} E / \mathrm{d} x_{0}$ which is treated as a variable in equation 2 . This probability is computed from a parameterisation of the Landau distribution. The ratio $p / M$ is then obtained in an iterative way from $\mathrm{d} E / \mathrm{d} x_{0}$, assuming that the particle is singly charged, using a parameterisation of the Bethe-Bloch formula for the restricted energy loss [15] which includes corrections for apparatus effects. The value of $M$ is then calculated using the measured track momentum. The maximum measurable mass using this technique is beyond the limit set by the centre of mass energy.

Figure 1 (upper plot) shows the specific energy loss for positively charged tracks, determined in this way, plotted against the track momenta. Clear bands can be seen corresponding to pions, kaons, protons, deuterons and tritons. The lower plot shows the spectrum of masses assigned to the tracks using the procedure described above. The smooth curves show parabolic fits to the $\log _{10} M$ distributions (i.e. Gaussians) in each of the different mass peaks. Some deviations from Gaussian behaviour in the tails of the distributions are observed. The resolution is $\delta M / M \sim 7 \%$ as determined from the widths of the Gaussian fits. The log-likelihood method adopted here is found to have better mass resolution and leads to a more Gaussian-like distribution than the method used previously by H1 [16]. The particle type for each track is identified as the mass of the closest known particle.

\subsection{Background Determination}

The distributions of the $z$ vertex coordinate and the distance of closest approach (DCA) of the tracks to the beam line in the transverse plane are used to distinguish the tracks produced in photoproduction from those produced by interactions of the beams with residual gas in the beam pipe, termed beam-gas interactions, or from the secondary interactions of photoproduced

\footnotetext{
${ }^{2}$ Throughout the paper $\mathrm{d} E / \mathrm{d} x$ is given as the ratio of the specifi c energy loss of the track to that of a MIP.
} 
particles in the material of the beam pipe or the detector, termed material background. The beam-gas backgrounds are labelled $p G$ for proton-gas and $e G$ for electron-gas interactions.

Figure 2 shows the DCA distributions. Tracks from interactions of the beam particles contribute to the peaks at zero, whereas the material background gives rise to the observed smooth background distributions. The material background for positive tracks results mainly from secondary interactions with the material of the beam pipe or the other material before the CJC sensitive region. This can be seen in the upper plots of figure 2 where it leads to an approximately flat background with small peaks at DCA $\sim 4 \mathrm{~cm}$. For negative tracks, the material background is much smaller (see figure 2 lower plots). It arises mainly from protons and deuterons which are back-scattered from the calorimeters into the CJC. These albedo particles, which lie outside the main peak in figure 2, are delayed due to their extra distance of travel by times of about $6 \mathrm{~ns}$ relative to the arrival time of tracks coming directly from the photoproduction interaction vertex, as measured in the CJC. The selection of negative particles with masses greater than the proton mass is supplemented by the requirement that this delay be less than $4 \mathrm{~ns}$, which is observed to reduce such backgrounds by about a factor of two.

The number of particles corrected for the material background is obtained by subtracting the number in the sidebands of the DCA distribution $(1.5<|\mathrm{DCA}|<3.0 \mathrm{~cm})$, normalised to the width of the selected region around the peak, from the total number of particles in the peak region. This width is chosen to be $\pm 1.5 \mathrm{~cm}$ for $p$ and $\bar{p}$ and $\pm 0.5 \mathrm{~cm}$ for heavier particles for which the DCA resolution is better.

The beam gas background is measured most accurately by dividing the data into four event samples depending on the presence or absence of energy in the forward part of the LAr or in the backward direction in the SpaCal. The event sample with both forward and backward energy (labelled $\mathrm{C}_{11}$ and comprising $89.4 \%$ of the total), arises dominantly from photoproduction $(\gamma p)$ which produces a roughly uniform distribution of energy in the apparatus for the $W_{\gamma p}$ range of this measurement. The event sample with forward but no backward energy (labelled $\mathrm{C}_{10}$ and comprising $8.5 \%$ of the total) arises dominantly from $p G$ interactions, since protons interacting in the interaction region with a nearly stationary target produce mainly forward but little significant backward energy. The event sample with backward but no forward energy (labelled $\mathrm{C}_{01}$ and comprising $2.0 \%$ of the total), is enriched in $e G$ interactions for similar reasons. Only $0.1 \%$ of the events have neither forward nor backward calorimetric energy (sample $\mathrm{C}_{00}$ ).

The data are separated into the $\gamma p, p G$ and $e G$ components in each sample, $\mathrm{C}_{i k}$, by studying the $z$ vertex distributions (see figure 3 ). The following procedure is adopted for the separation, the results of which are shown in table 1 for the hard selected tracks. First, the number of $\gamma p$ and $p G(e G)$ tracks in the samples $\mathrm{C}_{10}\left(\mathrm{C}_{01}\right)$, for each particle type, is measured by fitting the $z$ vertex distributions to the sum of a Gaussian and a linear background. The integral of the Gaussian is taken to be the number of particles from $\gamma p$ interactions, $N_{10}^{\gamma p}\left(N_{01}^{\gamma p}\right)$, while the linear background determines the number of $p G(e G)$ tracks, $N_{10}^{p G}\left(N_{01}^{e G}\right)$, in the samples. Here the number of $e G(p G)$ events in the $\mathrm{C}_{10}\left(\mathrm{C}_{01}\right)$ sample is neglected. The number of $e G$ particles in the $\mathrm{C}_{11}$ sample is also negligible. When the distribution has no visible Gaussian shape (e.g. figure 3d), the value of $N_{i k}^{\gamma p}$ is so low that a measurement is impossible (the dashes in table 1). Second, the number of $p G$ particles in each $\mathrm{C}_{11}$ sample, $N_{11}^{p G}$, is obtained by assuming that the probability that a true $p G$ event has significant backward energy is small. Hence, the probability that a $p G$ event appears in the $\mathrm{C}_{11}$ sample is approximately the probability that random noise 
above threshold occurs in the SpaCal calorimeter in a $p G$ event and this should be independent of particle type. This probability is measured from the ratio of the numbers of events in the $\mathrm{C}_{11}$ and $\mathrm{C}_{10}$ samples for pure $p G$ events, i.e. samples which should have little contribution from photoproduction. Deuterons with $z$ vertex $|z|>20 \mathrm{~cm}$ and $\cos \theta>0$ are used for this, as is an independent sample of events with two identified protons and no identified anti-proton, which also shows no significant Gaussian shape in the $z$ vertex distribution. The two independent measurements give ratios which agree within errors, confirming the assumption, with a mean of $0.151 \pm 0.045$. The quantity $N_{11}^{p G}$ is obtained by multiplying $N_{10}^{p G}$ by this ratio. Finally, the number of $\gamma p$ particles in the $\mathrm{C}_{11}$ sample, $N_{11}^{\gamma p}$, is obtained by subtracting $N_{11}^{p G}$ from the total for this sample, $N_{11}$. The separation into components for the $\mathrm{C}_{00}$ sample is made using the probabilities $(\sim 0.02$, estimated from the numbers in table 1$)$ that the photoproduced events have zero energy in the forward and backward calorimeters.

\subsection{Track Efficiencies}

The apparatus is fully sensitive in the range $0.2<p_{T} / M<0.7$ and $|y|<0.4$. In order to derive cross sections, corrections for track efficiencies (labelled $\epsilon$ ) must be applied. These are shown in detail for anti-deuterons in table 2 in the measurement intervals of $p_{T} / M$. The efficiencies for anti-protons tend to be somewhat larger than those for anti-deuterons, since the soft selection is used and the secondary interaction cross section is smaller. The efficiencies listed in table 2 are defined as follows.

- $\epsilon_{\mathrm{d} E / \mathrm{d} x}$ is the correction for migrations across the limit $\log \mathrm{d} E / \mathrm{d} x>0.4$. This is assessed by studying the migrations of anti-protons, selected with the looser criterion $\log \mathrm{d} E / \mathrm{d} x>$ 0.3 .

- $\epsilon_{\phi}$ is a correction for a region of inefficiency in the CJC which developed during the data taking.

- $\epsilon_{c u t}$ is a correction for the loss of events outside the mass and DCA windows.

- $\epsilon_{\sigma}$ represents the corrections for the losses due to interactions in the material of the apparatus. This is extracted from the data using soft selected, identified tracks. The number of such tracks which are observed to interact in the material between CJC1 and CJC2 is extrapolated to account for the material between the interaction point and the CJC1. The corrections agree with estimates from known cross sections.

- $\epsilon_{\text {hit }}$ represents the track reconstruction efficiency which is determined by measuring the fraction of soft selected deuterons which enter the hard selection sample.

- $\epsilon_{\text {trig }}$ is the trigger efficiency which is determined by two independent methods for events containing anti-protons. These are assumed to have the same trigger efficiency as antideuterons. One method involves Monte Carlo studies and the other method uses comparisons of the number of events from the main trigger with those found by an independent monitor trigger. The two methods give consistent results.

- $\epsilon_{t a g}$ is the positron tagger acceptance. 
- $\epsilon_{N c h}$ is the correction for the loss of events due to the requirement that there be five or more tracks in each event. This is deduced by applying the known KNO scaling distribution [17], using a sample of events containing anti-protons which has an observed mean track multiplicity which matches that for events containing anti-deuterons.

- $\epsilon_{t 0}$ represents the correction for the losses of anti-deuterons outside the defined track timing interval.

- $\epsilon_{P h S p}$ is an estimate of the fraction of anti-deuterons lost outside the measurement region defined by the limits of $|\eta|<1.0$ and $|y|<0.4$.

The final row of table 2 gives the measured value of the differential cross section

$$
\frac{\mathrm{d} \sigma}{\mathrm{d}\left(\mathrm{p}_{\mathrm{T}} / \mathrm{M}\right)}=\frac{N}{\Delta\left(p_{T} / M\right) \mathcal{L} F} .
$$

where $N$ is the number of events in each measurement interval, $\Delta\left(p_{T} / M\right)$, corrected for all the efficiencies given in table $2, \mathcal{L}=5.53 \pm 0.11 \mathrm{pb}^{-1}$ is the integrated luminosity and $F=0.0136$ is the virtual photon flux per incident positron (for the details of the calculation see [18]). The first error quoted for the differential cross sections is statistical while the second is systematic, where the latter arises from the uncertainties in the efficiencies. All cross sections are quoted at the bin centres.

\section{Results}

\subsection{Search for Heavy Particles}

The observed particle mass spectra are shown in figure 4. The dashed curves show the material backgrounds deduced from the sideband subtraction method described in section 2.3. The mainly $p G$ sample, $\mathrm{C}_{10}$, contains 6 tracks which have reconstructed masses of more than $3 \mathrm{GeV}$. They each have specific energy losses which are approximately twice that expected for a MIP. Visual inspection shows that these are overlapping tracks, probably due to relativistic particles which have been merged by the pattern recognition software, since the two tracks become visible at the ends of their trajectories. No such tracks are seen in the dominantly photoproduction sample, $\mathrm{C}_{11}$, which contains many more events. Since they are dominated by background, the $\mathrm{C}_{10}, \mathrm{C}_{01}$ and $\mathrm{C}_{00}$ samples are omitted from the search for heavy particles in photoproduction.

The observed deuterons and tritons (see table 1 and figure 4) are dominantly from the material background and upper limits on their photoproduction cross sections are derived from the observed numbers of events. These upper limits at the $95 \%$ confidence level, in the measurement range defined in section 2.5, are deduced to be 6.8 and $1.0 \mathrm{nb}$, respectively. No negative particles heavier than anti-deuterons and no positive particles heavier than tritons are observed. This allows an upper limit of $0.19 \mathrm{nb}$ at the $95 \%$ confidence level to be set on the photoproduction cross section for any such particle type in the same kinematic range. These cross section limits are derived assuming the same efficiencies as those for anti-deuterons given below. 


\subsection{The Anti-deuteron Cross Section}

A clear signal is seen in figure 4 , consisting of a total of 45 anti-deuterons with an estimated material background of $1.0 \pm 0.5$. The inclusive cross sections are measured using the 35 particles in the sensitive range defined in section 2.5 for all samples combined. Only two of the anti-deuterons are not in the $\mathrm{C}_{11}$ sample (both in $\mathrm{C}_{10}$ ). This number is compatible with that expected from the probability that a photoproduction event has small backward energy $(\sim 2 \%)$.

The total cross section for anti-deuteron production is found to be $2.7 \pm 0.5 \pm 0.2 \mathrm{nb}$ in the kinematic range defined in section 2.5 , by summing all the differential cross sections in the final row of table 2 . The ratio of the number of anti-deuterons to anti-protons in the range $|y|<0.4$ and $0.3<p_{T} / M<0.7$ is measured to be $(5.0 \pm 1.0 \pm 0.5) \cdot 10^{-4}$. The lower limit in $p_{T} / M$ is higher for the ratio than for the anti-deuteron cross section measurement in order to avoid the uncertainties associated with the large corrections to the anti-proton rates at low momentum.

The inclusive anti-deuteron invariant cross section is given by:

$$
\gamma \frac{\mathrm{d}^{3} \sigma}{\mathrm{d}^{3}(\mathrm{p} / \mathrm{M})}=M^{2} E \frac{\mathrm{d}^{3} \sigma}{\mathrm{d}^{3} \mathrm{p}}=\frac{1}{2 \pi\left(p_{T} / M\right) \Delta y} \frac{\mathrm{d} \sigma}{\mathrm{d}\left(\mathrm{p}_{\mathrm{T}} / \mathrm{M}\right)}
$$

where $\gamma=E / M, \mathrm{~d} \sigma / \mathrm{d}\left(\mathrm{p}_{\mathrm{T}} / \mathrm{M}\right)$ are the differential cross sections given in the final row of table 2 and $\Delta y=0.8$ is the rapidity range of the measurement. Figure 5 and table 3 show the measurements of this invariant cross section, normalised to the relevant total cross section, taken to be $165 \pm 11 \mu \mathrm{b}$ for photoproduction [18], as a function of $p_{T} / M$. Figure 5 also shows the measured ratio of the corrected numbers of anti-deuterons to anti-protons versus $p_{T} / M$.

\subsection{Comparison with Other Measurements}

Measurements of the normalised invariant anti-deuteron cross section and the ratio of the antideuteron to anti-proton production rates performed by other high energy experiments in the central region are also shown in figure 5. The normalised cross section and ratio measurements obtained in $p p$ collisions [5,6] and the photoproduction results described here are in good agreement, suggesting that the processes whereby anti-deuterons are formed are similar in $p p$ and $\gamma p$ interactions. The data on $\mathrm{Au}-\mathrm{Au}$ collisions show a ratio which is slightly larger than that in $p p$ collisions and photoproduction. However, the cross section for anti-deuteron production normalised to the total cross section in Au-Au collisions is over two orders of magnitude larger than that in photoproduction or $p p$ collisions, reflecting the more copious production of anti-nucleons in heavy ion collisions.

The coalescence parameter $B_{2}$ is derived by rearranging equation 1 to obtain

$$
B_{2}=\frac{\frac{1}{\sigma} \frac{E_{\bar{d}} \mathrm{~d}^{3} \sigma_{\overline{\mathrm{d}}}}{\mathrm{d}^{3} \mathrm{P}}}{\left(\frac{1}{\sigma} \frac{E_{\bar{p}} \mathrm{~d}^{3} \sigma_{\overline{\mathrm{p}}}}{\mathrm{d}^{3} \mathrm{p}}\right)^{2}}=\frac{M_{\bar{p}}^{4}}{M_{\bar{d}}^{2}} \frac{R^{2}}{\left(\frac{1}{\sigma} \frac{\gamma_{\bar{d}} \mathrm{~d}^{3} \sigma_{\overline{\mathrm{d}}}\left(\mathrm{P} / \mathrm{M}_{\overline{\mathrm{d}}}\right)}{}\right)} .
$$

Here, $M_{\bar{p}}$ and $M_{\bar{d}}$ are the masses of the $\bar{p}$ and $\bar{d}$ and $\sigma, \sigma_{\bar{p}}$ and $\sigma_{\bar{d}}$ are the total interaction

cross section and the partial cross sections for $\bar{p}$ and $\bar{d}$ production, respectively, as defined 
in equation 1 with $A=2$ for $\bar{d}$ production. The quantity, $R$, is the measured ratio of the number of anti-deuterons to anti-protons from direct production in each bin of $p_{T} / M$, corrected for anti-protons formed remotely from the source by weak decays. In the coalescence model, only anti-nucleons produced directly from the source can form anti-deuterons. The number of $\bar{p}$ from direct production is taken to be $78 \pm 8 \%$ of the number observed. This is estimated using the PYTHIA Monte Carlo [19], the accuracy being determined by the uncertainty in the strangeness suppression factor in this model. Hence, there is an overall theoretical uncertainty of about $20 \%$ in the determination of $B_{2}$. The weak decay correction is somewhat larger for heavy ion collisions [7] which is thought to be due to enhanced strangeness production [20].

Figure 6 and table 3 show the parameter $B_{2}$, computed according to equation 5 from the data presented here. This quantity is calculated for the ISR data in $[5,6]$, and is presented in figure 6 as a function of $p_{T} / M$. The average value in photoproduction is determined from the data presented here to be $B_{2}=0.010 \pm 0.002 \pm 0.001 \pm 0.002$, where the first uncertainty is statistical, the second systematic from the errors in the efficiencies and the third the theoretical error from the weak decay correction. Figure 7 shows the mean value of $B_{2}$ in photoproduction, compared with the mean $B_{2}$ values obtained from the ISR $p p$ data and the data of a variety of other experiments as a function of centre-of-mass energy. The value of $B_{2}$ measured in photoproduction at $W_{\gamma p}=200 \mathrm{GeV}$ is similar in magnitude to the values deduced at lower centre-of-mass energies in $p p$ and $p A$ interactions (labelled "elementary" in figure 7). However, this value of $B_{2}$ is over an order of magnitude larger than that observed in Au-Au collisions at RHIC at a similar nucleon-nucleon centre-of-mass energy. Comparison of the heavy ion data with the data from more elementary targets shows that this discrepancy grows with centreof-mass energy (see figure 7). To illustrate the difference between light and heavy colliding particles, the heavy ion data in figure 7 are restricted to very heavy ions. They are also restricted, at centre-of-mass energy below $50 \mathrm{GeV}$, to measurements of inclusive deuteron and proton production to avoid threshold effects in anti-deuteron production. The Bevelac data, at which energy the $A$ dependence is weak, are the Ne-Au measurements of [21]. The AGS data are the $\mathrm{Au}-\mathrm{Pt}$ measurements of the E886 experiment [22], the SPS data are the $\mathrm{Pb}-\mathrm{Pb}$ measurements of NA44 [23] and NA52 [24] and the RHIC data are from the Au-Au measurements of the STAR Collaboration [7]. The "elementary" data are the $p A$ data of $[21,22,25]$, the $p p$ data at the ISR $[5,6]$ and the photoproduction data presented here.

In the coalescence model for heavy ion collisions, the parameter $B_{2}$, varies inversely with both the volume of the fireball at thermal freeze-out and the absolute rate of anti-nucleon production when both the spatial and momentum dependence are included in the model [2]. The $\bar{d}$ to $\bar{p}$ ratio is observed in $p p$ and $\gamma p$ collisions to be close to that in Au-Au collisions, yet there is a much larger anti-nucleon production rate, and a smaller value of $B_{2}$ in $\mathrm{Au}-\mathrm{Au}$ collisions. These facts can be reconciled in the coalescence model if the size of the fireball at thermal freeze-out in $p p$ and $\gamma p$ collisions is much smaller than that in Au-Au collisions.

\section{Conclusions}

A search for heavy charged particles is made in photoproduction at HERA and anti-deuterons are observed at $\left\langle W_{\gamma p}\right\rangle=200 \mathrm{GeV}$. Upper limits at the $95 \%$ confidence level on the production 
cross sections for any type of positive particles heavier than tritons or negative particles heavier than anti-deuterons are set at $0.19 \mathrm{nb}$ in the kinematic range $|y|<0.4$ and $0.2<p_{T} / M<0.7$. The total cross section for anti-deuteron photoproduction is measured to be $2.7 \pm 0.5 \pm 0.2 \mathrm{nb}$ in the same kinematic range and the ratio of the number of anti-deuterons to anti-protons is measured to be $(5.0 \pm 1.0 \pm 0.5) \cdot 10^{-4}$ in the range $|y|<0.4$ and $0.3<p_{T} / M<0.7$. The transverse momentum dependence of the normalised invariant cross section for anti-deuteron production is found to be compatible with that measured in central $p p$ interactions at a centreof-mass energy of $53 \mathrm{GeV}$. The production rate per event of anti-deuterons in photoproduction is found to be over two orders of magnitude less than that observed in Au-Au collisions at RHIC, although the ratio of anti-deuterons to anti-protons is only slightly smaller. The coalescence model parameter $B_{2}$ is extracted in photoproduction and shown to be similar to that deduced from central high energy $p p$ data and lower energy $p A$ data. Averaging over the measurement range of $p_{T} / M, B_{2}$ is measured in photoproduction at $W_{\gamma p}=200 \mathrm{GeV}$ to be $0.010 \pm 0.002$ (stat.) \pm 0.001 (sys.) \pm 0.002 (theory) $\mathrm{GeV}^{2}$. This value is much larger than that observed in $\mathrm{Au}-\mathrm{Au}$ collisions at RHIC at a similar nucleon-nucleon centre-of-mass energy. This difference between heavy ion and elementary particle collisions is reduced as the centre-of-mass energy decreases. These observations can be understood within the framework of the coalescence model if the interaction volume at thermal freeze-out in $\gamma p$ and $p p$ collisions is much smaller than that in $\mathrm{Au}-\mathrm{Au}$ collisions at a centre-of-mass energy of $200 \mathrm{GeV}$.

\section{Acknowledgments}

We are grateful to the HERA machine group whose outstanding efforts have made this experiment possible. We thank the engineers and technicians for their work in constructing and now maintaining the $\mathrm{H} 1$ detector, our funding agencies for financial support, the DESY technical staff for continual assistance and the DESY directorate for support and for the hospitality which they extend to the non-DESY members of the collaboration.

\section{References}

[1] R.P. Duperray, K.V. Protasov and A.Yu. Voronin, Eur. Phys. J. A16 (2003) 27 [nuclth/0209078].

[2] R. Scheibl and U. Heinz, Phys. Rev. C59 (1999) 1585 [nucl/th/9809092];

A. Polleri, R. Mattiello, I.N. Mishustin and J.P. Bondorf, Phys. Lett. B473 (2000) 193 [nucl-th/9904012].

[3] S.T. Butler and C.A. Pearson, Phys. Rev. 129 (1963) 836;

A. Schwarzschild and C. Zupancic, Phys. Rev. 129 (1963) 854;

H.H. Gutbrod et al., Phys. Rev. Lett. 37 (1976) 667;

H. Sato and K. Yazaki, Phys. Lett. B98 (1981) 153;

L.P. Csernai and J.I. Kapusta, Phys. Rep. 131 (1985) 223.

[4] See figure 39.4, Particle Data Group, K. Hagiwara et al., Phys. Rev. 66 (2002) 1. 
[5] B. Alper et al., Phys. Lett. B46 (1973) 265.

[6] W.M. Gibson et al., Lett. Nuov. Cim. 21 (1978) 189.

[7] STAR Collaboration, C. Adler et al., Phys. Rev. Lett. 87 (2001) 262301-1, [nuclex/0108022].

[8] For a review of the lower energy data see A.G. Hansen, Ph.D. Thesis (Niels Bohr Institute) available at http://www.nbi.dk/na44/publications.html.

[9] V.V. Abramov et al., Sov. J. Nucl. Phys. 45 (1987) 845;

CHLM collaboration, M.G. Albrow et al., Nucl. Phys. B97 (1975) 189.

[10] V.V. Abramov et al., reference [9];

F. Binon et al., Phys. Lett. B30 (1969) 510;

Yu.M. Antipov et al., Phys. Lett. B34 (1969) 164;

J.W. Cronin et al., Phys. Rev. D11 (1975) 3105;

A. Aoki et al., Phys. Rev. Lett. 69 (1992) 2345;

E864 Collaboration, T.A. Armstrong et al., Phys. Rev. C59 (1999) 2699 [nuclex/9811002].

[11] ARGUS Collaboration, H. Albrecht et al., Phys. Lett. B236 (1990) 102.

[12] H1 Collaboration, I. Abt et al., Nucl. Instr. and Meth. A386 (1997) 310 and ibid. A386 (1997) 348.

[13] G.P. Eliseev, V.K. Kosmachevskii and V.A. Lubimov, Doklady Akad. Nauk SSSR 90 (1953) 995;

A.I. Alikhanov, V.A. Lubimov and G.P. Eliseev, CERN Symposium on High Energy Accelerator and Pion Physics, CERN, Geneva (1956).

[14] For a review see W. Blum and L. Rolandi, "Particle Detection with Drift Chambers", published by Springer-Verlag 1993.

[15] See article 26.2.4, Particle Data Group, K. Hagiwara et al., Phys. Rev. 66 (2002) 1.

[16] J. Steinhart, Ph.D. Thesis, Univerität Hamburg, available from http://wwwh1.desy.de/publications/theses_list.html.

[17] Z. Koba, H.B. Nielsen and P. Olesen, Nucl. Phys. B40 (1972) 317.

[18] H1 Collaboration, S. Aid et al., Z. Phys. C69 (1995) 27 [hep-ex/9509001]; ZEUS Collaboration, S. Chekanov et al., Nucl. Phys. B627 (2002) 3 [hep-ex/0202034].

[19] T. Sjöstrand et al., Comp. Phys. Commun. 135 (2001) 238 [hep-ph/0010017].

[20] D. Hardtke, private communication.

[21] S. Nagamiya et al., Phys. Rev. C24 (1981) 971.

[22] E886 Collaboration, G. Diebold et al., Phys. Rev. C48 (1993) 2984. 
[23] NA44 Collaboration, I. G. Bearden et al., Eur. Phys. J. C23 (2002) 237.

[24] NA52 Collaboration, G. Appelquist et al., Phys. Lett. B376 (1996) 245.

[25] NA44 Collaboration, H. Boggild et al., Nucl. Phys. A590 (1995) 483c. 

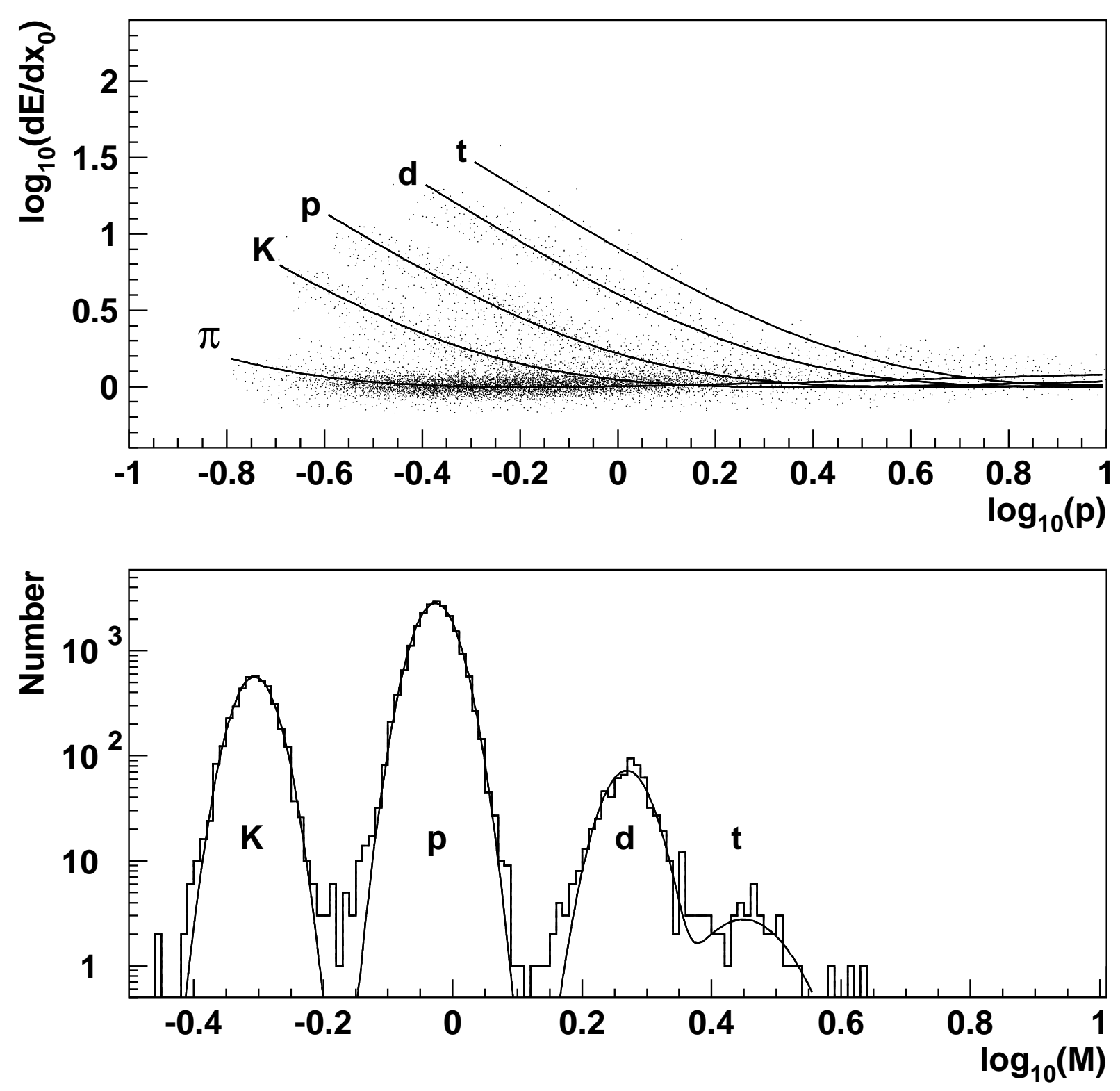

Figure 1: Upper plot - the observed specific ionisation energy loss, $\mathrm{d} E / \mathrm{d} x$, (normalised to that from a MIP), obtained from the log-likelihood method, versus track momentum (in $\mathrm{GeV}$ ) for a sample of positively charged tracks from the hard selection (before application of the specific energy loss cut, see text). The smooth curves show the expected mean specific energy loss for the different particle species. Lower plot - the spectrum of masses (in $\mathrm{GeV}$ ) for $\log _{10} \mathrm{~d} E / \mathrm{d} x>$ 0.4 , deduced as described in the text. The smooth curves represent Gaussian fits to each peak. 

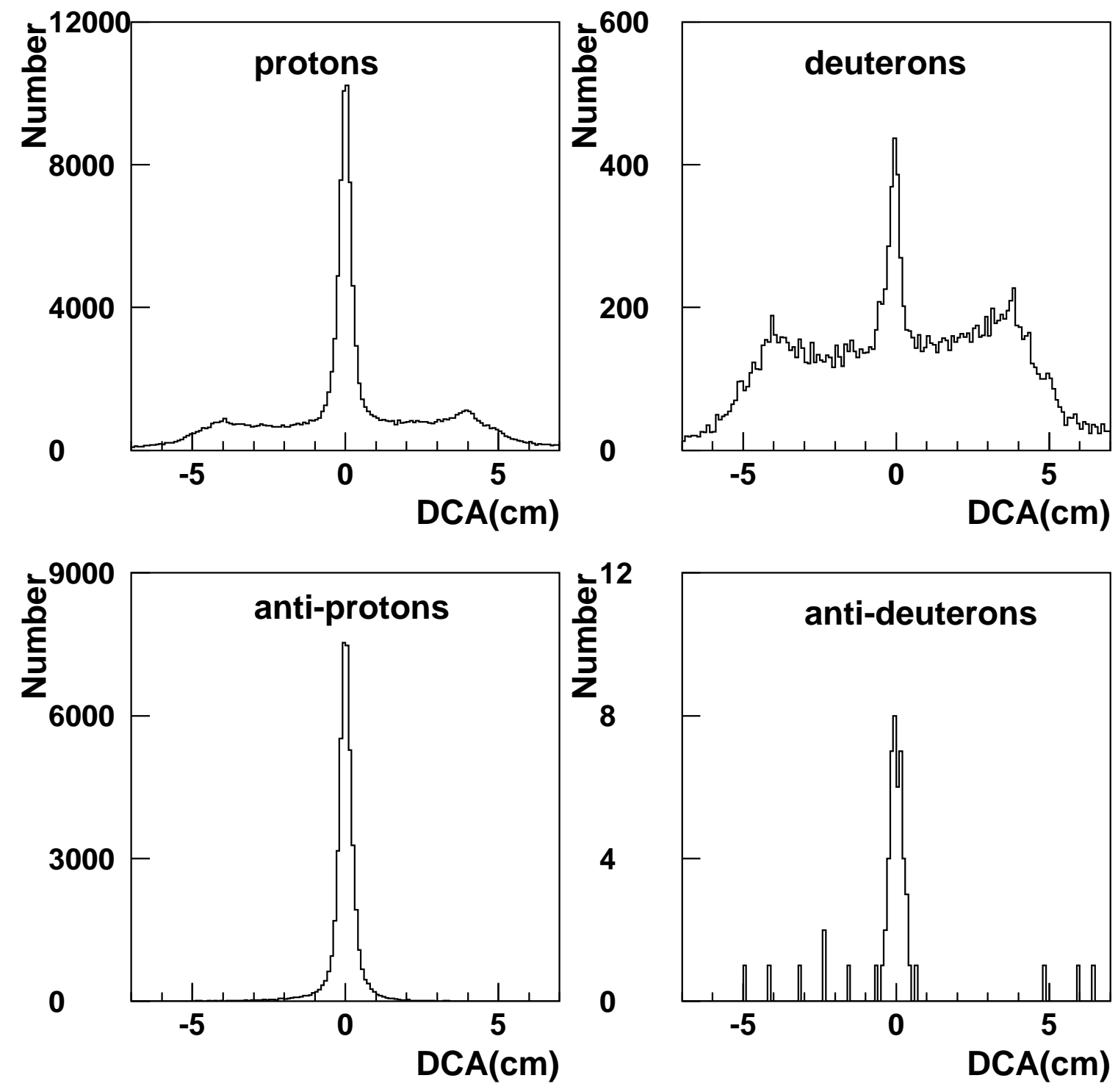

Figure 2: The distribution of the distance of closest approach (DCA) of the track to the event vertex in the plane transverse to the beam direction for $p, d, \bar{p}$ and $\bar{d}$ candidates with momenta larger than $0.5 \mathrm{GeV}$ from the hard selection and before any track timing cuts. The peak at zero from beam induced events sits on the material background which is much smaller for $\bar{p}, \bar{d}$ than for $p, d$. 

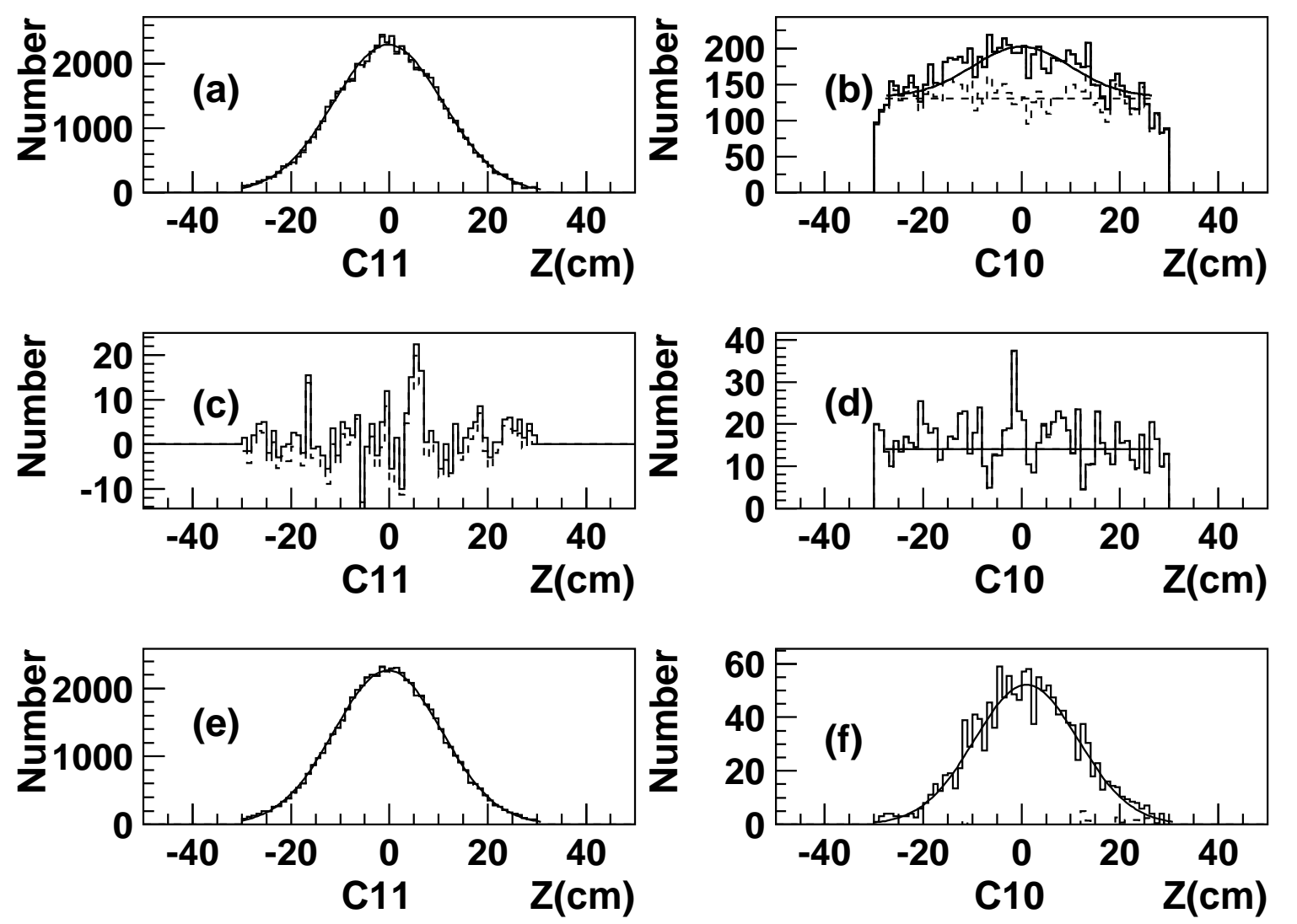

$\overline{\mathbf{p}}$
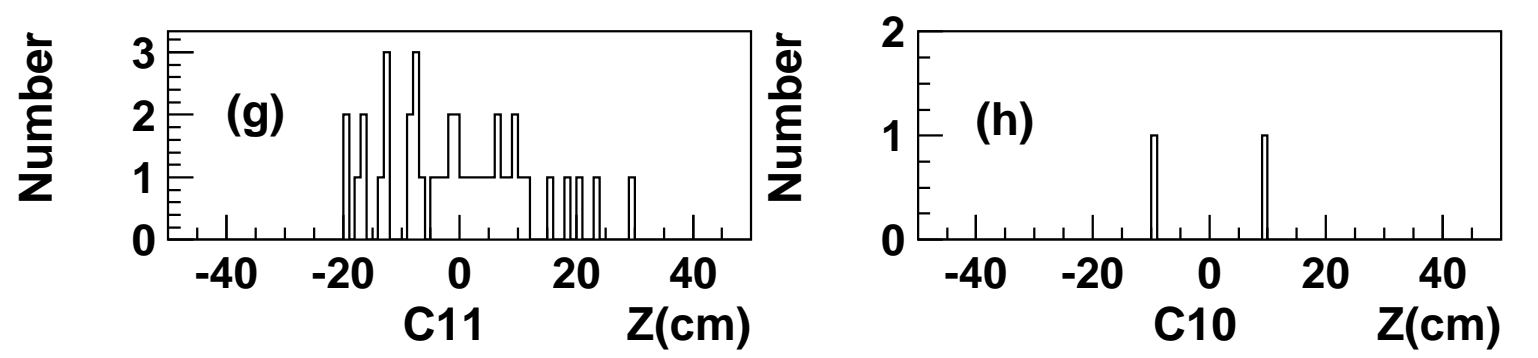

$\overline{\mathbf{d}}$

Figure 3: The $z$ vertex distributions for identified protons $(\mathrm{a}, \mathrm{b})$, deuterons $(\mathrm{c}, \mathrm{d})$, anti-protons $(\mathrm{e}, \mathrm{f})$ and anti-deuterons $(\mathrm{g}, \mathrm{h})$ in the hard selected samples after correction for the material backgrounds. The $\mathrm{C}_{11}$ samples (figures a,c,e,g) are dominated by photoproduction events and the $\mathrm{C}_{10}$ sample (figures b,d,f) are dominated by $p G$ events. The solid curves (figures a,b,c,d) show the fits of a Gaussian distribution (expected from photoproduction) and a linear background (expected from $p G$ interactions). The lack of background and the relatively small number of events in (f) show that there is little $\bar{p}$ production in $p G$ events. The number of $\bar{d}$ events in (h) is compatible with that expected from the $\gamma p$ contamination of $\mathrm{C}_{10}$ sample. The lack of background in (a) and (e) shows that there are few $p G$ events in the $\mathrm{C}_{11}$ sample. The dashed histograms represent the background after subtraction of the fitted (b) or calculated (c,f) Gaussians. 

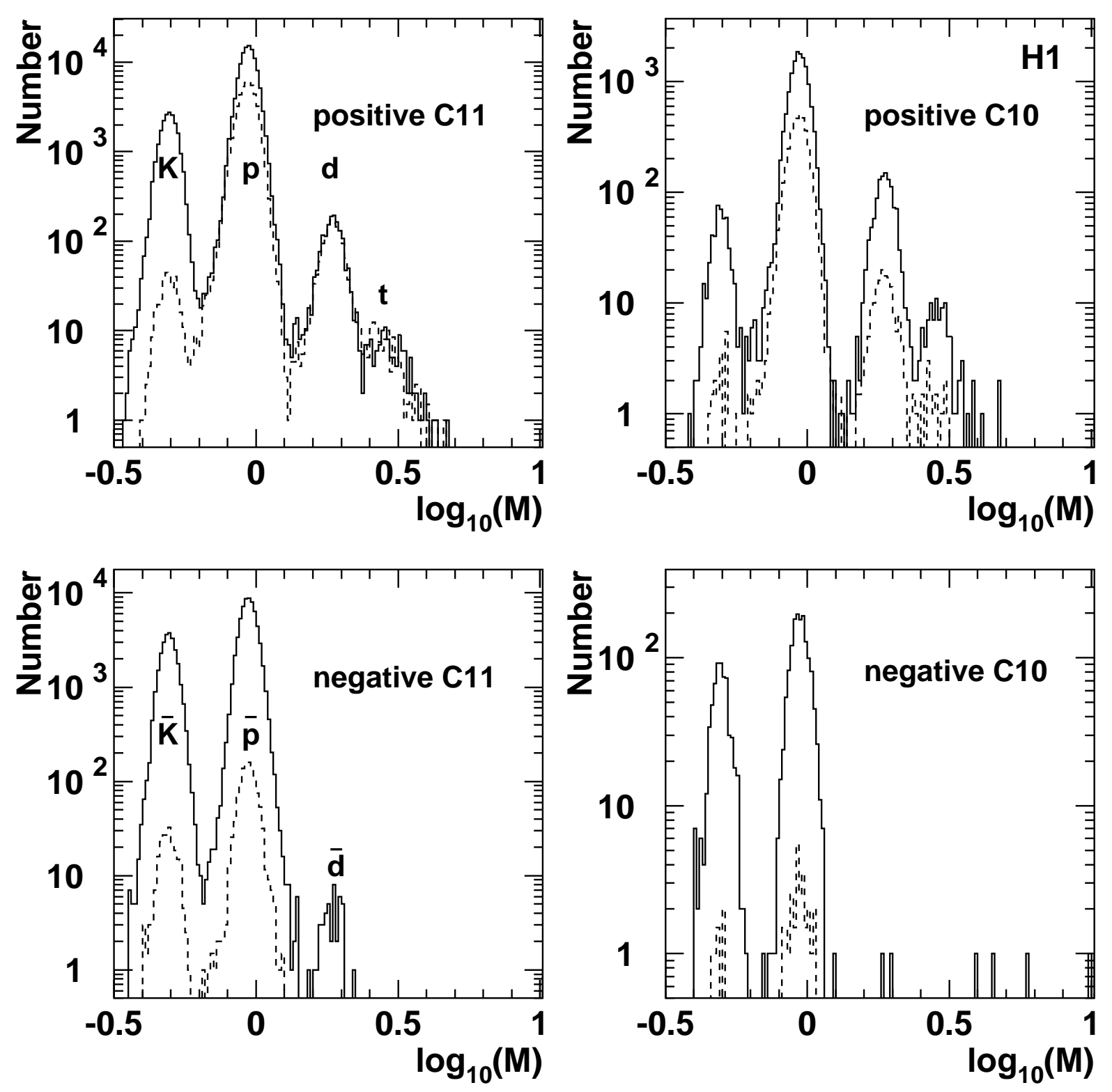

Figure 4: The mass spectra (with $M$ in $\mathrm{GeV}$ ) for positive and negative particles in the hard selected sample for the mainly photoproduction $\left(\mathrm{C}_{11}\right)$ and mainly $p G\left(\mathrm{C}_{10}\right)$ samples. The dashed lines show the material background deduced from the sideband subtraction method. 

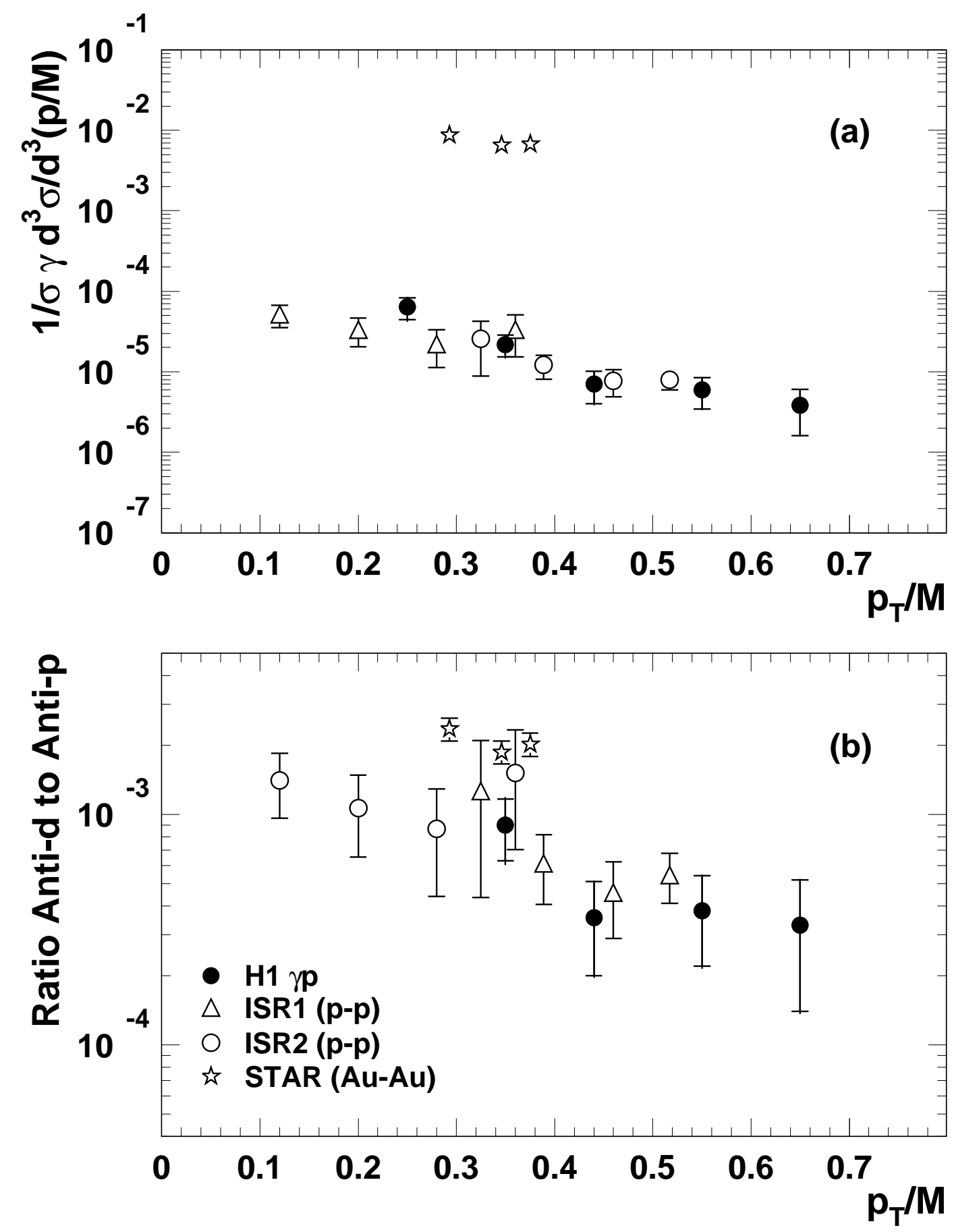

Figure 5: a) The measured invariant cross section (normalised to the relevant total cross section) for inclusive $\bar{d}$ production, compared with the $p p$ and Au-Au data. b) The measured $\bar{d}$ to $\bar{p}$ production ratio as a function of $p_{T} / M$ (solid points) compared with $p p$ data from the ISR at $\sqrt{s}=53 \mathrm{GeV}$ in the central region [5,6] and RHIC data on Au-Au collisions [7]. The inner error bars on the $\mathrm{H} 1$ data indicate the statistical and the outer the total uncertainties. 


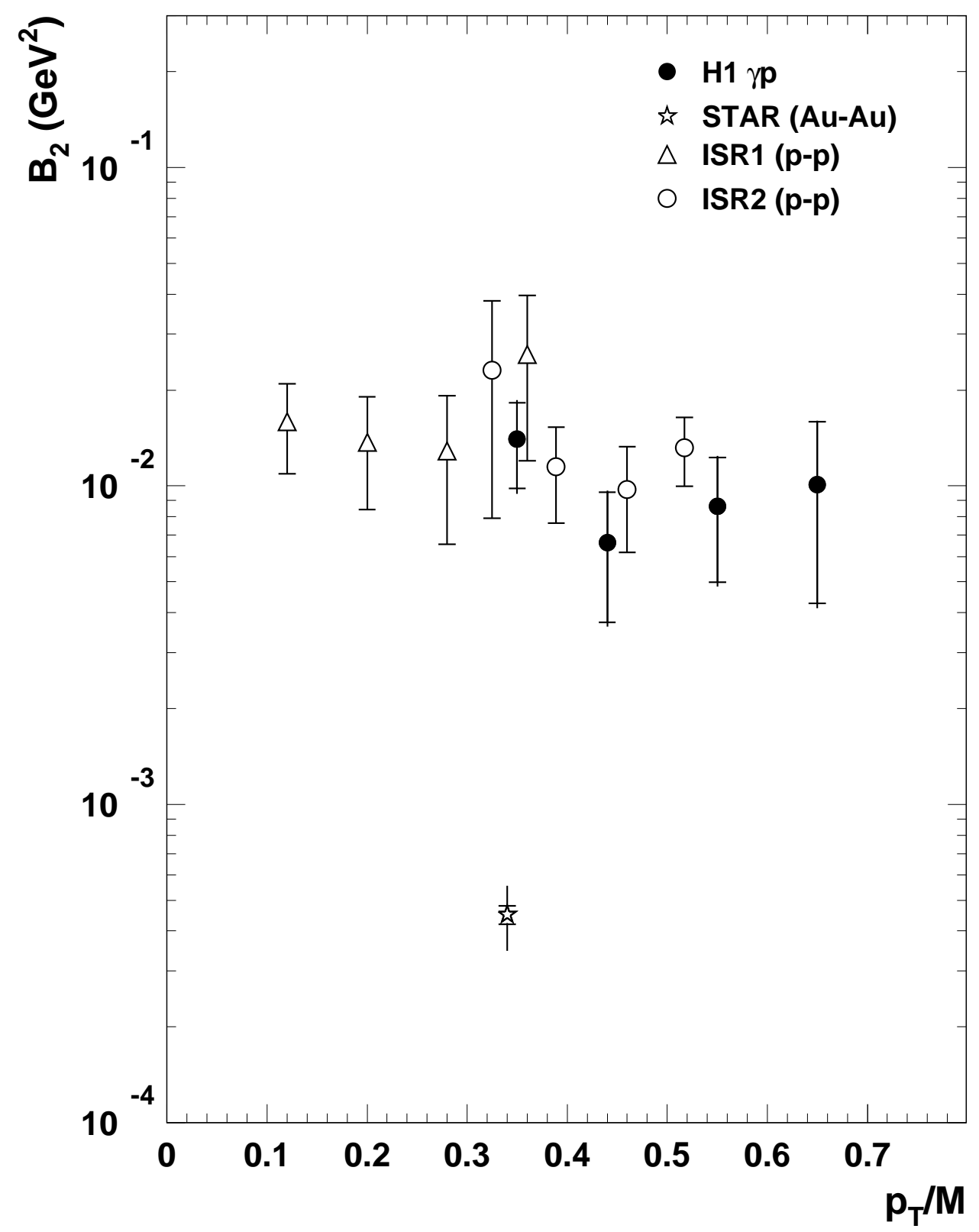

Figure 6: The $p_{T} / M$ dependence of the parameter $B_{2}$ for $\gamma p$ (this experiment), $p p[5,6]$ and Au-Au interactions [7]. The values of $B_{2}$ for the $p p$ data are deduced from the measured cross sections $[5,6]$. There is a theoretical uncertainty of about $20 \%$ (not shown) in the determination of $B_{2}$ for the photoproduction and $p p$ data, which arises from the calculation of the fraction of anti-protons produced directly (see text). The inner error bars on the H1 data indicate the statistical and the outer the total uncertainties. 


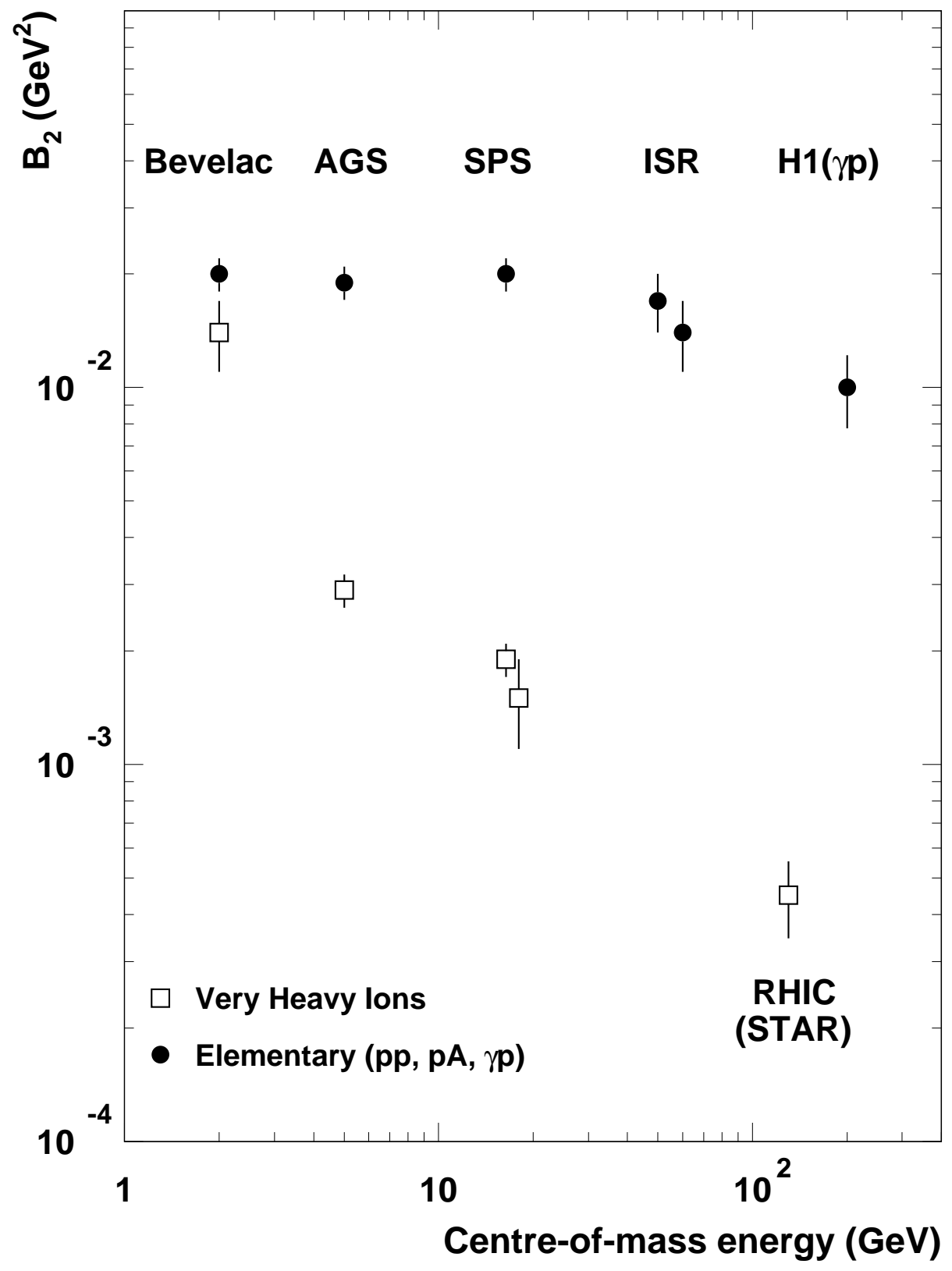

Figure 7: The dependence of $B_{2}$ on centre-of-mass energy for very heavy ion collisions (open squares) and interactions of more elementary particles (closed circles). The heavy ion data are the $\mathrm{Ne}-\mathrm{Au}$ data at the Bevelac [21], the E886 Au-Pt data at the AGS [22], the Pb-Pb data of NA44 [23] and NA52 [24] at the SPS and the Au-Au data of STAR [7] at RHIC. The "elementary" data are the $p A$ data of $[21,22,25]$, the $p p$ data at the ISR $[5,6]$ and the photoproduction data presented here. In the latter the error bar represents the total uncertainty i.e. the sum in quadrature of the systematic and statistical uncertainties. 
Table 1: The numbers $\left(N_{i k}^{\gamma p, p G, e G}\right)$ of photoproduction $(\gamma p)$ and beam gas $(p G, e G)$ hard selected tracks $\left(\log _{10} \mathrm{~d} E / \mathrm{d} x>0.4\right)$ in the four event samples (see text) for the different particle types. The right hand column gives the observed total number of identified tracks in each category after subtraction of the material background. The quoted errors are the statistical uncertainties.

\begin{tabular}{|c|c|c|c|c|c|}
\hline & Sample & $N_{i k}^{\gamma p}$ & $N_{i k}^{p G}$ & $N_{i k}^{e G}$ & $N_{i k}$ \\
\hline$p$ & $\mathrm{C} 11$ & $62883 \pm 510$ & $1177 \pm 350$ & - & $64060 \pm 370$ \\
$p$ & $\mathrm{C} 10$ & $1858 \pm 520$ & $7794 \pm 510$ & - & $9652 \pm 124$ \\
$p$ & $\mathrm{C} 01$ & $1153 \pm 86$ & - & $322 \pm 71$ & $1475 \pm 51$ \\
$p$ & $\mathrm{C} 00$ & $25 \pm 10$ & $80 \pm 45$ & - & $105 \pm 11$ \\
\hline$p$ & $N^{\gamma p, p G, e G}$ & $65919 \pm 733$ & $9051 \pm 620$ & $322 \pm 71$ & $75292 \pm 394$ \\
\hline \hline$d$ & $\mathrm{C} 11$ & $15 \pm 56$ & $137 \pm 41$ & - & $152 \pm 44$ \\
$d$ & $\mathrm{C} 10$ & $0 \pm 1$ & $908 \pm 38$ & - & $908 \pm 38$ \\
$d$ & $\mathrm{C} 01$ & - & - & $33 \pm 7$ & $33 \pm 7$ \\
$d$ & $\mathrm{C} 00$ & - & $5 \pm 2$ & - & $5 \pm 2$ \\
\hline$d$ & $N^{\gamma p, p G, e G}$ & $15 \pm 56$ & $1050 \pm 60$ & $33 \pm 7$ & $1098 \pm 60$ \\
\hline \hline$t$ & $\mathrm{C} 11$ & $1 \pm 9$ & $10 \pm 3$ & - & $11 \pm 9$ \\
$t$ & $\mathrm{C} 10$ & - & $68 \pm 9$ & - & $68 \pm 9$ \\
$t$ & $\mathrm{C} 01$ & - & - & - & - \\
$t$ & $\mathrm{C} 00$ & - & - & - & - \\
\hline$t$ & $N^{\gamma p, p G, e G}$ & $1 \pm 9$ & $78 \pm 10$ & - & $79 \pm 13$ \\
\hline \hline $\bar{p}$ & $\mathrm{C} 11$ & $61949 \pm 252$ & $1 \pm 9$ & - & $61950 \pm 252$ \\
$\bar{p}$ & $\mathrm{C} 10$ & $1425 \pm 71$ & $5 \pm 60$ & - & $1430 \pm 38$ \\
$\bar{p}$ & $\mathrm{C} 01$ & $1363 \pm 70$ & - & $-15 \pm 60$ & $1348 \pm 37$ \\
$\bar{p}$ & $\mathrm{C} 00$ & $21 \pm 5$ & - & - & $21 \pm 5$ \\
\hline $\bar{p}$ & $N^{\gamma p, p G, e G}$ & $64758 \pm 270$ & $6 \pm 60$ & $-15 \pm 60$ & $64749 \pm 255$ \\
\hline \hline$d$ & $\mathrm{C} 11$ & $43 \pm 7$ & - & - & $43 \pm 7$ \\
$\bar{d}$ & $\mathrm{C} 10$ & $2 \pm 1.4$ & - & - & $2 \pm 1.4$ \\
$\bar{d}$ & $\mathrm{C} 01$ & - & - & - & - \\
$\bar{d}$ & $\mathrm{C} 00$ & - & - & - & - \\
\hline$d$ & $N^{\gamma p, p G, e G}$ & $45 \pm 7$ & - & - & $45 \pm 7$ \\
\hline & & & & & \\
\hline
\end{tabular}


Table 2: The observed number of events, track and event efficiencies and the differential cross section for $\bar{d}$ production (see section 2.5 ).

\begin{tabular}{|c|c|c|c|c|c|}
\hline$P_{T} / M$ & 0.25 & 0.35 & 0.45 & 0.55 & 0.65 \\
\hline$N_{\bar{d}}^{\text {obs }}$ & 11 & 11 & 5 & 5 & 3 \\
$\epsilon_{(\mathrm{d} E / \mathrm{d} x)}$ & 1.0 & 1.0 & 1.0 & $0.97 \pm 0.01$ & $0.75 \pm 0.03$ \\
$\epsilon_{\phi}$ & $0.97 \pm 0.01$ & $0.97 \pm 0.01$ & $0.97 \pm 0.01$ & $0.97 \pm 0.01$ & $0.97 \pm 0.01$ \\
$\epsilon_{\text {cut }}$ & $0.83 \pm 0.05$ & $0.96 \pm 0.02$ & 1.0 & 1.0 & 1.0 \\
$\epsilon_{\sigma}$ & $0.80 \pm 0.07$ & $0.82 \pm 0.07$ & $0.85 \pm 0.07$ & $0.86 \pm 0.07$ & $0.87 \pm 0.07$ \\
$\epsilon_{\text {hit }}$ & $0.67 \pm 0.04$ & $0.86 \pm 0.03$ & $0.86 \pm 0.03$ & $0.86 \pm 0.03$ & $0.86 \pm 0.03$ \\
$\epsilon_{\text {trig }}$ & $0.82 \pm 0.04$ & $0.82 \pm 0.04$ & $0.82 \pm 0.04$ & $0.82 \pm 0.04$ & $0.82 \pm 0.04$ \\
$\epsilon_{\text {tag }}$ & $0.46 \pm 0.02$ & $0.46 \pm 0.02$ & $0.46 \pm 0.02$ & $0.46 \pm 0.02$ & $0.46 \pm 0.02$ \\
$\epsilon_{N c h}$ & $0.96 \pm 0.02$ & $0.96 \pm 0.02$ & $0.96 \pm 0.02$ & $0.96 \pm 0.02$ & $0.96 \pm 0.02$ \\
$\epsilon_{t 0}$ & $0.95 \pm 0.02$ & $0.97 \pm 0.01$ & $0.98 \pm 0.01$ & $0.98 \pm 0.01$ & $0.98 \pm 0.01$ \\
$\epsilon_{P h S p}$ & $0.75 \pm 0.06$ & $0.96 \pm 0.03$ & 1.0 & 1.0 & 1.0 \\
\hline$\frac{\mathrm{d} \sigma}{\mathrm{dp} \mathrm{M} / \mathrm{M}}(n b)$ & $13.1 \pm 3.9 \pm 2.2$ & $6.6 \pm 2.0 \pm 0.8$ & $2.7 \pm 1.1 \pm 0.3$ & $2.7 \pm 1.1 \pm 0.3$ & $2.1 \pm 1.2 \pm 0.2$ \\
\hline
\end{tabular}

Table 3: The measured values of the invariant $\bar{d}$ production cross sections, $\bar{d}$ to $\bar{p}$ ratios and coalescence parameter, $B_{2}$. The first error is the statistical and the second error the systematic uncertainty.

\begin{tabular}{|c|c|c|c|c|}
\hline$p_{T} / M$ & $\gamma \frac{\mathrm{d}^{3} \sigma_{\overline{\mathrm{d}}}}{\mathrm{d}^{3}\left(\mathrm{p} / \mathrm{M}_{\overline{\mathrm{d}}}\right)}(\mathrm{nb})$ & $\begin{array}{c}R_{\text {meas }}=\frac{N_{\bar{d}}}{N_{\bar{p}}} \cdot 10^{4} \\
\text { measured value }\end{array}$ & $\begin{array}{c}R_{\text {corr }} \cdot 10^{4} \\
\text { weak decay corrected }\end{array}$ & $B_{2}\left(\mathrm{GeV}^{2}\right)$ \\
\hline 0.25 & $10.5 \pm 3.2 \pm 1.7$ & - & - & - \\
\hline 0.35 & $3.6 \pm 1.1 \pm 0.5$ & $9.0 \pm 2.7 \pm 1.2$ & $11.8 \pm 3.6 \pm 1.6$ & $0.015 \pm 0.004 \pm 0.002$ \\
\hline 0.45 & $1.2 \pm 0.5 \pm 0.1$ & $3.6 \pm 1.6 \pm 0.4$ & $4.7 \pm 2.1 \pm 0.5$ & $0.007 \pm 0.003 \pm 0.001$ \\
\hline 0.55 & $1.0 \pm 0.4 \pm 0.1$ & $3.8 \pm 1.6 \pm 0.5$ & $4.8 \pm 2.0 \pm 0.6$ & $0.009 \pm 0.004 \pm 0.001$ \\
\hline 0.65 & $0.6 \pm 0.4 \pm 0.1$ & $3.3 \pm 1.9 \pm 0.4$ & $4.2 \pm 2.4 \pm 0.5$ & $0.010 \pm 0.006 \pm 0.001$ \\
\hline
\end{tabular}

